\title{
COMPARISON BETWEEN AIRBORNE LIDAR DATA AND DIGITAL IMAGE MATCHING FOR SURFACE RECONSTRUCTION
}

\author{
A. C. Carrilho ${ }^{1}$ *, R. C. dos $\operatorname{Santos}^{1}$, G. G. Pessoa ${ }^{1}$, M. Galo ${ }^{1,2}$ \\ ${ }^{1}$ Graduate Program in Cartographic Sciences - PPGCC, São Paulo State University - UNESP, \\ Presidente Prudente, São Paulo, Brazil - andre.carrilho@unesp.br, renato.cesar@unesp.br, guilherme.g.pessoa@unesp.br \\ ${ }^{2}$ Dept. of Cartography, São Paulo State University - UNESP, \\ Presidente Prudente, São Paulo, Brazil - mauricio.galo@unesp.br
}

KEY WORDS: Surface Reconstruction, Airborne Laser Scanning, Digital Image Matching, Point Cloud.

\begin{abstract}
:
Nowadays some aerial surveying projects (both manned or not) might integrate imagery and ranging sensor technology, thus allowing both the reconstruction of the surface from airborne laser scanning (ALS) and from the Photogrammetric pipeline through digital image matching (DIM). DIM algorithms have been continuously improved, therefore comparisons to other techniques such as ALS must be conducted. Despite the scientific community efforts, there are few evaluations regarding the extraction of building roofs using point clouds derived from both ALS and imagery. In this sense, this study provides a brief comparison between ALS and DIM point clouds and the accuracy that can be achieved in surface reconstruction and building roofs extraction. The experiments indicated that in some situations such as the building roof shape extraction, the accuracy is similar between these techniques, however, this is not valid for vertical accuracy, where larger differences were observed.
\end{abstract}

\section{INTRODUCTION}

In the past few decades, there have been an adoption trend of airborne surveying with integrated sensors such as digital aerial imagery and airborne laser scanning (ALS), for instance. According to Remondino et al. (2013) the main reason to adopt ALS systems over Photogrammetry during the early stages of the digital era was due to the lack of hardware and software necessary to achieve similar accuracy to ranging sensors when generating dense point clouds with digital image matching (DIM). Some drawbacks arose from this data acquisition approach, such as the increase in the project's cost due to the necessity of more sensors to be integrated in the platform. Recently, some studies attempted to use both ALS and DIM point clouds towards a better topographic reconstruction (Mandlburger et al., 2017).

The technological advances enabled improvements on both the hardware computational capacity and the algorithms that runs on it. Since the late 1990's, there were several breakthroughs from the computer vision scientific community which, together with the computational power of general-purpose graphic processing units (GPGPU), made feasible some techniques such as the structure from motion (SfM) and multi-view stereo (MVS). Haala (2009) emphasizes that both feature and/or intensity-based matching for automatic aerial triangulation was available on commercial software from more than two decades back, i.e., since 1989.

Two classes of methods are intrinsically related to the SfM pipeline: the sparse and dense image matching. The first class of methods had a significant leap with the scale invariant feature transform (SIFT) (Lowe, 2004) and subsequently by similar techniques such as the speeded-up robust features (SURF) (Bay et al., 2008), for instance. Those methods, together with the random sample consensus (RANSAC), provided great improvements and automation on image matching and, consequently, on image orientation tasks and $3 \mathrm{D}$ reconstruction from images. The second class of methods (dense matching) can be tracked down to approaches such as the vertical line locus (VLL), that can be considered in different modes, as single stereo (Helava, 1988), multi-view and in enhanced multi-view (Zhang et al., 2018). However, the most basic algorithms are likely to fail under some conditions, since they rely on template matching (area-based matching). Image matching is considered as an illconditioning problem (Heipke, 1996), since the solution may exist, not exist (due to occlusion) or be ambiguous (in homogeneous surfaces and/or repeated patterns). The leap in computational power allowed the development of more robust techniques such as the semi-global matching (SGM), which addresses those problems by introducing penalties on the cost function minimization procedure (Hirschmüller, 2008; Ressl et al., 2016). According to Haala (2009), the SGM consist of a pixelwise matching technique based on mutual information which applies an approximation of a global smoothness constraint.

As mentioned earlier, aerial surveying projects (both manned or not) can integrate imagery and ranging sensor technology, thus allowing both the reconstruction of the surface from the ALS and the Photogrammetric pipeline. Despite the scientific community efforts, there are few evaluations regarding the surface reconstruction and extraction of building roofs using point clouds derived from both ALS and imagery.

\subsection{ALS versus DIM}

The accuracy of ALS derived point clouds depends on two principal factors: the distance measurement accuracy and the quality in which the platform trajectory can be retrieved. This second factor is also important for the direct georeferenced imagery on SfM derived point clouds, however, the main aspects that contribute to the accuracy, in this case, lies in the geometrical properties of the camera and the block of images, that is, overlapping percentage and baseline-to-height ratio, for instance. Haala (2009) emphasizes that the geometric accuracy of the DIM

\footnotetext{
* Corresponding author
} 
is influenced by the quality of the reconstructed image geometry during the bundle block adjustment.

According to Mandlburger et al. (2017), the typical point spacing, as well as the expected height precision for DIM point clouds, can be down to image GSD. In this case, it is assumed that a robust matching technique is applied, and the proper geometric conditions of the block are met. The height measured by ALS can be even more precise, as of $2-3 \mathrm{~cm}$ on modern systems. Ressl et al. (2016) report that nowadays, the GSD is typically in the range of $5-20 \mathrm{~cm}$, with the possibility of being even smaller with the usage of UAVs, i.e., lower flight heights.

\subsection{Related work}

Haala (2009) evaluated the surfaces reconstructed using the software "MATCH-T DSM" over the German Society for Photogrammetry, Remote Sensing and Geoinformation (DGPF) dataset which resulted on several digital surface models (DSM). According to Ressel et al. (2016), the "MATCH-T DSM" implements the SGM algorithm under the cost-based matching (CBM) terminology. Haala (2009) adopted an ALS point cloud as a reference, and the comparison results made clear that image matching is more error-prone, mainly if there are moving objects during the image acquisition. Haala (2011) has also verified the feasibility of multiple stereo matching on the SGM algorithm, i.e., using the multi-view stereo (MVS) to compute the disparity maps. Depending on the forward and side overlaps, the redundancy on the point correspondence can benefit the geometric accuracy of the surface, as well as allowing an efficient quality analysis. From these studies, the author mentions that although less accurate, the image matching point clouds can be used for several standard applications with sufficient quality.

A comparative discussion over LiDAR and SGM for DSM generation was published by Gehrke et al. (2012). In this study the authors presented a dense matching approach based on stereo images captured with line scanners. The authors emphasize some advantages of the SGM technique over ALS, such as the lower cost and that the orthoimages can be generated from the same data set. From this comparison the authors concluded that the SGM-derived point cloud is in accordance with the ALS one, except in vegetated areas.

A similar study was conducted by Rahmayudi and Rizaldy (2016), where the authors compared DTM generated from image matching and ALS. It was understood that point clouds derived from image matching are prone to error on post-processing such as filtering in ALS. Although both techniques might generate point clouds with outliers, ALS is likely to present a lower quantity of them in general. Also, ALS is more interesting even on densely vegetated areas, since the pulse penetration in the canopy can reach deeper levels (even the ground surface in some cases, depending on how sparse the tree canopy is).

Maltezos and Ioannidis (2015) conducted an evaluation of the automatic detection of building roofs using point cloud derived from ALS and image. In this study the authors conclude that, although DIM is a reliable technique, ALS still provides better accuracy. Especially in the DIM context, Dall'Asta and Roncella (2014) performed a comparison between local and global matching algorithms for surface reconstruction. As expected from the results of image matching (Heipke, 1996), local matching techniques are less reliable on homogeneous surfaces or on repetitive patterns than global approaches. This emphasizes the dependency on texture features in order to achieve good performance.

Exploring the current trend of deep learning applications, Politz and Sester (2018) tested Convolutional Neural Networks (CNN) on both ALS and DIM data towards a better semantic segmentation. The point cloud data needed to be converted to a raster representation, which eliminates the density factor. The experiments performed by these authors showed that the ALS was able to outperform the DIM, since the best network achieved an overall accuracy of $96 \%$ and $83 \%$, respectively.

From this discussion, the method that produces the best positional accuracy might be unclear, since the literature is not in accordance. However, several studies indicate that both data sources are comparable and useful for some applications. In this sense, our study provides a comparison between ALS and DIM point clouds by assessing the absolute positional accuracy achieved with both techniques. In the following assessment, the characteristics unique to a single technique are disregarded, such as laser beam penetration in the vegetation canopy, for instance.

\section{MATERIALS}

The data used in this study comes from the UNESP Photogrammetric Data Set (Tommaselli et al., 2018), which made available the aerial imagery and ALS point clouds (Table 1). The flights were conducted in 2014, and the region of interest (highlighted in Figure 1) selected for this study comprises the central area of the UNESP campus in Presidente Prudente/Brazil.

\begin{tabular}{ll|ll}
\hline \multicolumn{2}{c|}{ Aerial imaging characteristics } & \multicolumn{2}{c}{ ALS characteristics } \\
\hline Camera manufacturer & Phase One & ALS manufacturer & Riegl \\
Camera model & iXA 180 & ALS model & LMS-Q680i \\
Operation range (min, max) & $100 \mathrm{~m}, 10000 \mathrm{~m}$ & Operantion range (min, max) & $30 \mathrm{~m}, 2000 \mathrm{~m}$ \\
Nominal focal length* & $55 \mathrm{~mm}$ & Laser beam divergence & $\leq 0.5 \mathrm{mrad}$ \\
Pixel size & $5.2 \mu \mathrm{m}$ & Scanning mechanism & rotating polygon mirror \\
Radiometric resolution & $12 \mathrm{bit}$ & Scan pattern & parallel scan lines \\
Spectral bands & R-G-B & Scan angle range & $\pm 30^{\circ}$ \\
CCD format (mm) & $53.70 \mathrm{x} 40.40$ & Scan speed & $10-200$ lines $/ \mathrm{sec}$ \\
CCD format (px) & $10328 \times 7760$ & Measurement rate & up to $266 \mathrm{kHz}$ \\
Forward motion compensation & by TDI & Nominal accuracy ** & $20 \mathrm{~mm}$ \\
Average flying height & $1300 \mathrm{~m}$ & Average flying height & $550 \mathrm{~m}$ \\
Average GSD & $12 \mathrm{~cm}$ & Average density & 12 points $/ \mathrm{m}^{2}$ \\
\hline * selected from 28-240 mm range & \multicolumn{3}{c}{$* *$ at 250 m distance } \\
\multicolumn{1}{c}{ Table 1 - Characteristics of both data and equipment used to acquire the point clouds. }
\end{tabular}




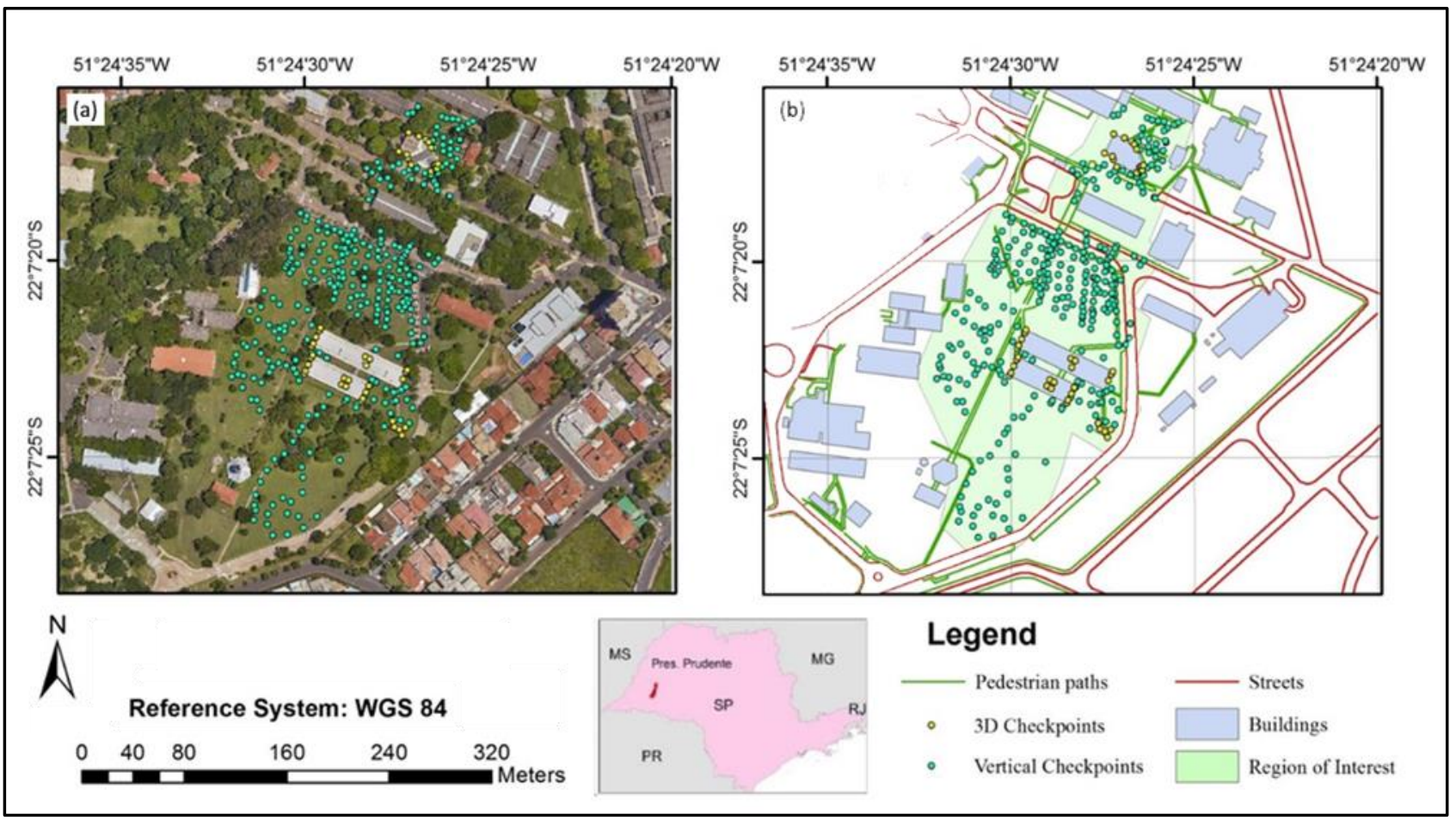

Figure 1 - Study area, the region of interest and checkpoints survey.

There are several software for SfM data processing: Agisoft Metashape, Pix4Dmapper, and nFrames SURE, for instance. However, despite parameter tuning effects, these software provide similar results as reported by Alidoost and Arefi (2017). For the analyses considered in this paper, the SfM pipeline was performed in Agisoft Metashape version 1.5.3 (formerly known as Photoscan), whereas both ALS and DIM point clouds were manipulated and visualized in CloudCompare version 2.10. The vertical accuracy assessment was performed using the rapidlasso LAStools library and ESRI ArcGIS version 10.7.

In total, 303 vertical checkpoints were collected in the region of interest (shown in Figure 1) using a couple of double frequency Topcon Hiper Lite GNSS receivers. The coordinates were determined with approximately $1 \mathrm{~cm}$ precision using RTK positioning. All vertical checkpoints were collected on the ground surface, most of them over grass. This assessment procedure forgoes the necessity of artificial targets to be placed before the flight since a close approximation of the vertical coordinates is already enough to compute the height difference. Additionally, 48 checkpoints were collected in building roof corners on the region of interest. This data collection was conducted with a reflector-less Topcon GTS-239W total station in two closed traverse surveys. Each checkpoint was measured from at least two different stations to ensure redundancy.

\section{EXPERIMENTS}

\subsection{Absolute positional accuracy}

Two experiments were conducted to assess the absolute accuracy of both ALS and DIM point clouds, and their results are presented in Table 2. In experiment I, the vertical accuracy was determined for both point clouds. In total, 303 vertical checkpoints were considered, being used the lascontrol tool from rapidlasso LAStools. This tool computes the height difference between the checkpoint and the expected height at the same position in the point cloud. To derive the expected height, a triangular irregular network (TIN) mesh is computed over the point cloud, which is used to interpolate the height. In experiment II a simpler and straightforward approach was adopted. In this strategy, the 3D Euclidian distance from each of the 48 checkpoints to their respective closest ones in both point clouds was computed.

\begin{tabular}{c|c|c|c|c}
\cline { 2 - 5 } \multicolumn{2}{c|}{} & \multicolumn{2}{c|}{$\begin{array}{c}\text { Experiment I } \\
\text { (only Z) }\end{array}$} & \multicolumn{2}{c}{$\begin{array}{c}\text { Experiment II } \\
\text { (3D distance) }\end{array}$} \\
\hline $\begin{array}{c}\text { Data } \\
\text { source }\end{array}$ & $\begin{array}{c}\text { Mean } \\
(\mathbf{c m})\end{array}$ & $\begin{array}{c}\text { Std. Dev. } \\
(\mathbf{c m})\end{array}$ & $\begin{array}{c}\text { Mean } \\
(\mathbf{c m})\end{array}$ & $\begin{array}{c}\text { Std. Dev. } \\
(\mathbf{c m})\end{array}$ \\
\hline ALS & 7.05 & 3.45 & 31.28 & 33.42 \\
DIM & -34.85 & 23.28 & 49.19 & 35.34 \\
\hline
\end{tabular}

Table 2 - Absolute accuracy assessment.

It is important to observe that the ALS point cloud was acquired at less than half the flight height of the images $(550 \mathrm{~m}$ other than $1300 \mathrm{~m}$ ), thus, it is expected to have better accuracy. As can be seen from the experiment I (shown in Table 2), the ALS system was able to produce a point cloud with better vertical accuracy than the DIM. The spatial distribution of vertical errors is better visualized in Figure 2. Apart from displaying a narrower vertical error range, the ALS point cloud appears to have a better distribution (similar to a Gaussian) of those errors as shown in the histograms in Figure 2.

Most of the vertical errors in the ALS point cloud are in accordance with manufacturer claims (Table 1). A similar note can be derived for the DIM point cloud, in which most of the vertical errors are within 3 GSD. Observing the Figure $2 b$ and comparing it to Figure 1a, it can be noted a positional correlation between the vertical errors and the amount of texture information in the images. In order to investigate this effect, two small regions were selected (one homogeneous and another heterogeneous) and had their vertical errors compared to the color histograms generated from the digital numbers (DN) in each band $(R, G, B)$, as shown in Figure 3. 

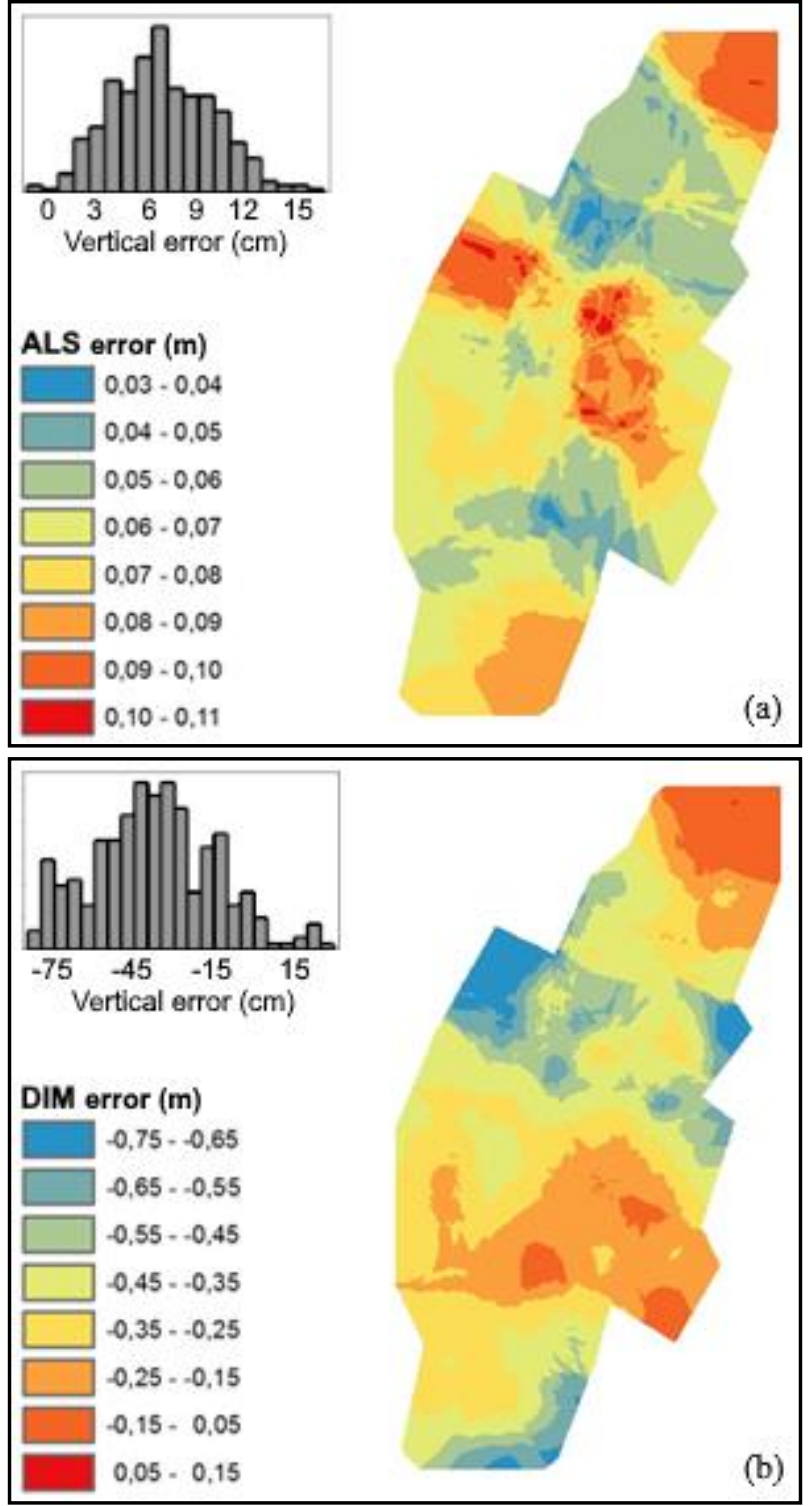

Figure 2 - Vertical error assessment: Kriging results and respective histogram. ALS (a) and DIM (b) point clouds.

Pure grass regions such as in Figure 3a are more likely to yield poor matching accuracy, therefore an increase in vertical error due to the lack of texture. Otherwise, if the region contains at least some features as in Figure 3b, the vertical error tends to minimize.

Observing the results from experiment II and accounting for the flight height difference, it can be noted that both techniques provide similar accuracy. However, the ALS will be a better option in most cases if the vertical accuracy is crucial, as in some engineering tasks, for instance. It is important to highlight that this observation refers to an automatic DIM approach and the results might be different if a human operator performs the measurements.

\subsection{Building roof shape extraction}

To perform the building extraction assessment, a reference shape is needed. The Presidente Prudente dataset reference polygons building roof outlines were derived using a manual image restitution process, following the Photogrammetric pipeline, performed in the ERDAS IMAGINE system.
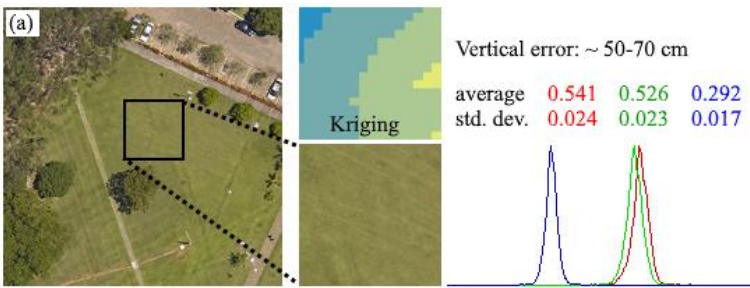
$\begin{array}{llll}\text { average } & 0.541 & 0.526 & 0.292 \\ \text { std. dev. } & 0.024 & 0.023 & 0.017\end{array}$
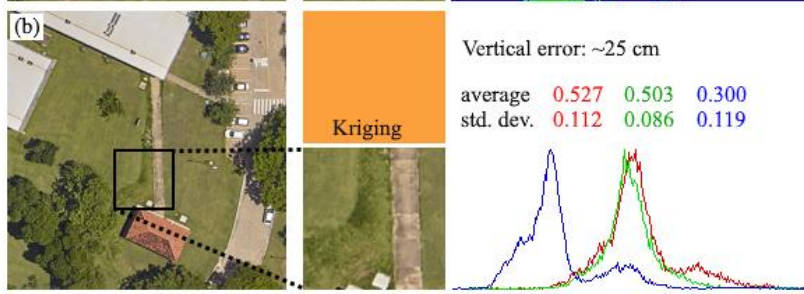

Figure 3 - Influence of texture information on DIM vertical accuracy for 2 areas: homogeneous (a) and heterogeneous (b). The average and standard deviation are in DN (digital numbers), normalized in the interval $[0,1]$ for each image band (R, G, B).

According to Potučkvá and Hofman (2016), there are several metrics available to perform the quality assessment of the extracted polygons that represent building roofs. This study will focus on the Polygons and Line Segments (PoLiS) metric proposed by Avbelj et al. (2015) and in F-score (Sokolova et al., 2006). Considering two polygons $A$ and $B$ with $q$ and $r$ vertices, respectively, the PoLiS metric is defined as:

$$
p(A, B)=\frac{1}{2 q} \sum_{a_{j} \in A} \min _{b \in \partial B}\left\|a_{j}-b\right\|+\frac{1}{2 r} \sum_{b_{k} \in B} \min _{a \in \partial A}\left\|b_{k}-a\right\|
$$

Other than distance-based metrics, the error matrix quantities can be adapted to evaluate the extraction accuracy as well. The true positives (TP), false positives (FP), true negatives (TN) and false negatives (FN) can be computed using the area of the objects of interest. The completeness and correctness are two metrics derived from the error matrix that are commonly used to evaluate remote sensing data. A third metric is the F-score, which can be computed as the weighted harmonic mean of the completeness and correctness, but can be also derived directly from the elementary quantities TP, FP and FN:

$$
\mathrm{F} \text {-score }=\frac{2 \mathrm{TP}}{2 \mathrm{TP}+\mathrm{FP}+\mathrm{FN}}
$$

The two selected metrics (PoLiS and F-score) have a distinct interpretation. When the extracted polygon is in agreement with the reference, it is expected that the PoLiS value is going to approach zero (since it is a distance measure), whereas the Fscore will converge towards one (as an accuracy rate).

Two buildings were selected to evaluate the building roof shape extraction (Figure 4). These two buildings are a laboratory and a coffee shop, respectively, both within the Unesp campus. The building boundaries were extracted from both ALS and DIM point clouds using the same method and then compared to the reference. As mentioned before, to apply the boundary extraction algorithm, the point cloud must be classified, and the building points identified. In this study, this pre-processing step was conducted using the following tools: lasground (with the "city" option enabled), lasheight and lasclassify. Finally, the lasboundary was applied to extract the building roof shapes, using the "disjoint" and "concavity 1" options enabled. 


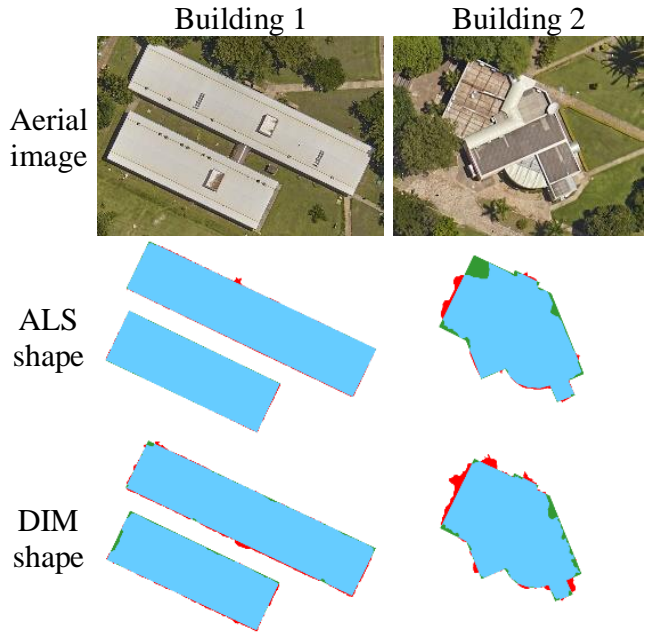

Figure 4. Building roof shape extraction quality assessment. Legend: TP $(\bullet), \mathrm{FN}(\bullet)$ and FP $(\bullet)$

The reference polygon vertex count for each building is presented in Table 3, and these values can be interpreted as indicators of geometrical complexity. A noticeable fact is that usually, the DIM results yield polygons with a bigger area than the ALS (Table 3). This also can be seen in Figure 4, but by the FP occurrences.

The F-score and PoLiS metrics are presented in Table 4, where it can be noted that in general, the ALS achieved a similar or better accuracy for these two buildings. The PoLiS distance computed for Building 1 also presents a considerable discrepancy between ALS and DIM.

\begin{tabular}{c|c|c|c|c}
\hline \multirow{2}{*}{ Building } & \multicolumn{3}{|c|}{ Area $\left(\mathbf{m}^{2}\right)$} & \multirow{2}{*}{$\begin{array}{c}\text { Number of } \\
\text { vertices }\end{array}$} \\
\cline { 2 - 4 } & Reference & ALS & DIM & \\
\hline $\mathbf{1}$ & 1974.05 & 1988.77 & 1990.85 & 8 \\
$\mathbf{2}$ & 507.86 & 487.94 & 516.75 & 19 \\
\hline
\end{tabular}

Table 3. Characteristics of the selected buildings.

Vegetation close to the building roofs can cause problems, as can be noted for Buildings 1 and 2 in Figure 3. In these cases, some of the FP occurrences are due to the incorrect classification of canopy near the roof edges. Objects such as antennas (Building 2) are also error sources, as they increase the geometrical complexity of the building roof.

\begin{tabular}{c|c|c|c|c}
\hline \multirow{2}{*}{ Building } & \multicolumn{2}{|c|}{ F-score } & \multicolumn{2}{c}{ PoLiS (m) } \\
\cline { 2 - 5 } & $\boldsymbol{A L S}$ & $\boldsymbol{D I M}$ & $\boldsymbol{A L S}$ & $\boldsymbol{D I M}$ \\
\hline $\mathbf{1}$ & 0.99 & 0.98 & 0.27 & 0.58 \\
$\mathbf{2}$ & 0.95 & 0.95 & 0.52 & 0.52 \\
\hline Average & 0.97 & 0.96 & 0.39 & 0.55 \\
\hline
\end{tabular}

Table 4. Building roof shape extraction results.

These results show that, although DIM provides a slightly less accurate building roof delineation when compared to ALS, it constitutes an alternative data source that can be used for several applications.

\section{CONCLUSIONS}

In summary, both data sources (ALS and DIM) were able to provide accurate point clouds. The advantage of ALS is that the equipment is robust to some characteristics of land cover that can be problematic on matching algorithms, such as the lack of texture information. In those situations, the ALS is expected to provide better vertical accuracy. Despite those cases, the two techniques can achieve comparable results.

Further research must be conducted to assess different aspects of ALS and DIM techniques. Besides, it is important to keep this discussion, comparison, and positional accuracy assessment up to date, as newer equipment and methods are in constant development. The DIM has great potential to improve as newer algorithms are developed.

\section{ACKNOWLEDGEMENTS}

The authors would like to thank Graduate Program on Cartographic Sciences from FCT-UNESP, Presidente Prudente-SP/Brazil; Sensormap Geotecnologia for providing the LiDAR data; São Paulo Research Foundation - FAPESP (grant 2019/05268-8) for supporting this research; National Council for Scientific and Technological Development - CNPq (grant 304189/2016-2); and Coordenação de Aperfeiçoamento de Pessoal de Nível Superior - CAPES (Finance Code 001).

\section{REFERENCES}

Alidoost, F. and Arefi, H. Comparison of UAS-based photogrammetry software for 3D point cloud generation: a survey over a historical site. ISPRS Annals of Photogrammetry, Remote Sensing and Spatial Information Sciences, IV-4/W4, pp. 55-61, 2017.

Avbelj, J.; Muller, R. and Bamler, R. A metric for polygon comparison and building extraction evaluation. IEEE Geoscience and Remote Sensing Letters, v. 12, n. 1, pp. 170-174, 2015.

Bay, H.; Tuytelaars, T. and Gool, L.V. SURF: speeded up robust features. Computer Vision and Image Understanding, v. 110, n. 3, pp. 346-359, 2008.

Bianco, S.; Ciocca, G. and Marelli, D. Evaluating the performance of structure from motion pipelines. Journal of Imaging, v. 4, n. 98, pp. 18, 2018.

Dall'Asta, E. and Roncella, R. A comparison of Semi-Global and Local dense matching algorithms for surface reconstruction. ISPRS Archives of the Photogrammetry, Remote Sensing and Spatial Information Sciences, XL, 5, pp. 187-194, 2014.

Gehrke, S.; Morin, K.; Downey, M.; Boehrer, N. and Fuchs, T. Semi-Global Matching: an alternative to LiDAR for DSM generation? ISPRS Archives of the Photogrammetry, Remote Sensing and Spatial Information Sciences, XXXVIII, 1, pp. 1-6, 2010.

Haala, N. Comeback of Digital Image Matching. Photogrammetric Week, pp. 289-301, 2009.

Haala, N. Multiray Photogrammetry and Dense Image Matching. Photogrammetric Week, pp. 185-195, 2011.

Haala, N.; Hastedt, H.; Wolf, K.; Ressl, C. and Baltrusch, S. Digital Photogrammetric Camera Evaluation - Generation of Digital Elevation Models. Photogrammetrie - Fernerkundung Geoinformation, v. 2, pp. 99-115, 2010.

Heipke, C. Overview of image matching techniques. In: OEEPE - Workshop on the application of Digital Photogrammetric Workstations. Lausanne, Switzerland: 1996. 
Helava, U. V. Object space least-squares correlation. Photogrammetric Eng. Remote Sensing, 54 (6), pp. 711-714, 1988.

Hirschmüller, H. Stereo processing by semi-global matching and mutual information. IEEE Transactions on Pattern Analysis and Machine Intelligence, v. 30, n. 2, pp. 328-341, 2008.

Lowe, D. G. Distinctive image features from scale-invariant keypoints. International Journal of Computer Vision, v. 60, n. 2, pp. 91-110, 2004.

Maltezos, E. and Ioannidis, C. Automatic detection of building points from LiDAR and dense image matching point clouds. ISPRS Annals of Photogrammetry, Remote Sensing and Spatial Information Sciences, II-3/W5, pp. 33-40, 2015.

Mandlburger, G.; Wenzel, K.; Sptzer, A.; Haala, N.; Glira, P. and Pfeifer, N. Improved topographic models via concurrent airborne LiDAR and Dense Image Matching. ISPRS Annals of Photogrammetry, Remote Sensing and Spatial Information Sciences, v. IV-2/W4, pp. 259-266, 2017.

Politz, F. and Sester, M. Exploring ALS and DIM data for semantic segmentation using CNNs. ISPRS Archives of the Photogrammetry, Remote Sensing and Spatial Information Sciences, XLII-1, pp. 347-354, 2018.

Potučkvá, M. and Hofman, P. Comparison of quality measures for building outline extraction. The Photogrammetric Record, v. 31, n. 154, pp. 193-209, 2016.

Rahmayudi, A. and Rizaldy, A. Comparison of semi automatic DTM from image matching with DTM from LiDAR. ISPRS Archives of the Photogrammetry, Remote Sensing and Spatial Information Sciences, XLI-B3, pp. 373-380, 2016.

Remondino, F.; Spera, M. G.; Nocerino, E.; Menna, F.; Nex, F. and Gonizzi-Barsanti, S. Dense image matching: comparisons and analyses. 2013 Digital Heritage International Congress, IEEE, pp. 47-54, 2013.

Ressl, C.; Brockmann, H.; Gottfried, K. and Pfeifer, N. Dense Image Matching vs. Airborne Laser Scanning - Comparison of two methods for deriving terrain models. Photogrammetrie, Fermerkundung, Geoinformation, pp. 57-73, 2016.

Sokolova, M.; Japkowicz, N. and Szpakowicz, S. Beyond accuracy, f-score and roc: a family of discriminant measures for performance evaluation. In: Proceedings of the AAAI'06 workshop on Evaluation Methods for Machine Learning. p. 2429, 2006.

Tommaselli, A. M. G.; Galo, M.; Reis, T. T.; Ruy, R. S.; Moraes, M. V. A. and Matricardi, W. V. Development and assessment of a data set containing frame images and dense airborne laser scanning point clouds. IEEE Geoscience and Remote Sensing Letters, v. 15, n. 2, pp. 192-196, 2018.

Zhang, K.; Sheng, Y.; Wang, M. and Fu, S. An enhanced multiview vertical line locus matching algorithm of object space ground primitives based on positioning consistency for aerial and space images. ISPRS Journal of Photogrammetry and Remote Sensing, 139, pp. 241-254, 2018. 